\title{
Risk Factors and Their Diagnostic Values for Ocular Metastases in Gastric Adenocarcinoma
}

\author{
Yue Chen ${ }^{1, *}$ \\ Yan-Chang Yang $\mathbb{D}^{2, *}$ \\ Li-Ying Tang ${ }^{3, *}$ \\ Qian-Min $\mathrm{Ge} \mathbb{D}^{4}$ \\ Wen-Qing Shi $\mathbb{D}^{1,4}$ \\ Ting Su $\mathbb{D}^{3,5}$ \\ Hui-Ye Shu',4 \\ Yi-Cong Pan ${ }^{1,4}$ \\ Rong-Bin Liang ${ }^{4}$ \\ Qiu-Yu Li ${ }^{4}$ \\ Yi Shao (iD) ${ }^{4}$ \\ 'Department of Dermatology, The Eighth \\ Affiliated Hospital of Sun Yat-sen \\ University, Shenzhen, Guangzhou, \\ 518033, People's Republic of China; \\ ${ }^{2}$ Department of Anesthesiology, \\ Nanchang University, Nanchang, Jiangxi, \\ 330006, People's Republic of China; \\ ${ }^{3}$ Department of Ophthalmology, \\ Zhongshan Hospital of Xiamen \\ University, Xiamen, Fujian Province, \\ 36II02, People's Republic of China; \\ ${ }^{4}$ Department of Geriatric Medicine and \\ Ophthalmology, The First Affiliated \\ Hospital of Nanchang University, \\ Nanchang, Jiangxi, 330006, People's \\ Republic of China; ${ }^{5}$ Department of \\ Ophthalmology, Massachusetts Eye and \\ Ear, Harvard Medical School, Boston, MA, \\ 02।14, USA
}

*These authors contributed equally to this work

Correspondence: $Y_{i}$ Shao

Department of Geriatric Medicine and Ophthalmology, The First Affiliated Hospital of Nanchang University, No. 17, YongWaiZheng Street, DongHu District, Nanchang, Jiangxi, 330006, People's

Republic of China

$\mathrm{Tel} / \mathrm{Fax}+86$ 79I-88692520

Email freebee99@I63.com
Objective: Gastric adenocarcinoma originates from the glands in the superficial layer or mucosa of the stomach. It is prone to metastases, of which ocular metastasis (OM) is rare, but once it occurs the disease is considered more serious. The aim of this study was to investigate the risk factors for $\mathrm{OM}$ in gastric adenocarcinoma.

Methods: Patients with gastric adenocarcinoma were recruited to this study between June 2003 and July 2019. Demographic data and serological indicators (SI) were compared between patients with and without $\mathrm{OM}$, and binary logistic regression was used to explore whether the relevant SI may be risk factors for OM of gastric adenocarcinoma. Receiver operating characteristic (ROC) curves were used to analyze different SIs for OM in gastric cancer patients.

Results: Chi-square tests showed significant between-groups difference in gender composition $(\mathrm{P}<0.05)$, but not in age or histological grade $(\mathrm{P}>0.05)$. $t$-test results showed that lowdensity lipoprotein (LDL) and carbohydrate antigen-724 (CA724) were significantly higher in patients with than without $\mathrm{OM}(\mathrm{P}<0.05)$. Binary logistic regression analysis showed that LDL was an independent risk factor for OM $(\mathrm{P}<0.001)$. ROC curve analysis showed that the areas under the curves (AUC) for LDL and CA724 were 0.903 and 0.913 respectively, with higher AUC for combined LDL and CA724 (0.934; $\mathrm{P}<0.001)$.

Conclusion: LDL and CA724 have value as predictors for OM in patients with gastric adenocarcinoma, with higher predictive value when these factors are combined.

Keywords: gastric adenocarcinoma, serological indicators, ocular metastases, risk factors, ROC curve

\section{Introduction}

Gastric cancer (GC) is a common gastrointestinal cancer $^{1}$ with varied incidence globally. In recent years, the incidence rates of gastric cancer in the United States, Canada and Britain have been decreasing, but it remains the seventh highest cause of death in the United States. In Japan, although the incidence rate has declined, GC is still the most common malignant tumor. ${ }^{2}$ About $95 \%$ of GC is gastric adenocarcinoma, originating from the most superficial or mucosal gland of the stomach. ${ }^{3}$ In addition, mucosa associated lymphoid tissue (MALT) lymphoma originates from gastric lymphoid tissue and leiomyosarcoma originates from muscles around the mucosa. ${ }^{4}$

This paper mainly focuses on gastric adenocarcinoma. The immutable risk factors of gastric adenocarcinoma include gender, age, family history and race. Modifiable factors include smoking, radiation, Helicobacter pylori infection, high salt intake and low intake of fruits and vegetables. ${ }^{1}$ Due to improvements in public 
health, nutrition, hygiene level and health awareness, as well as the optimization and adjustment of diet structure, the incidence of gastric adenocarcinoma related to geographical area and diet has decreased significantly. However, it is worth noting that the infection rate of Helicobacter pylori has increased significantly, causing an estimated $65 \%$ to $80 \%$ of gastric adenocarcinoma cases and has become one of the main factors contributing to the incidence of gastric adenocarcinoma. ${ }^{5}$ The mechanism of this effect remains unknown, but may be related to the indirect effect of inflammation caused by Helicobacter pylori on gastric epithelial cells and the direct effect of bacteria on epithelial cells. Alternatively, Helicobacter pylori may directly regulate the function of epithelial cells through bacterial factors (such as CagA), and both of these mechanisms are thought to interact in the development of $\mathrm{GC}^{6}$

In the early stage, most patients with gastric adenocarcinoma have no clear clinical manifestations, but do report abdominal discomfort, pantothenic nausea, belching, and loss of appetite. In the late stage, the symptoms may include fever, significant weight loss, chest tightness, dysphagia, and even hemoptysis. ${ }^{7}$ Since early symptoms are not specific, patients with recurrent upper abdominal discomfort or high-risk factors should be examined thoroughly to ensure early diagnosis, timely treatment and better survival rate. In recent years, due to the continuous progress of medical technology, the pathogenesis of gastric cancer has become clearer, and the prevention and treatment strategies are constantly improving. At present, treatment methods for gastric adenocarcinoma include surgery, radiotherapy, chemotherapy and immunotherapy. ${ }^{8}$ Due to the high proportion of lymph node metastasis in early gastric cancer, early measures are of great significance to patients' prognosis. ${ }^{9}$ However, radiotherapy and chemotherapy can reduce mortality in patients with advanced gastric cancer who cannot tolerate surgery or for whom surgery is unlikely to be beneficial.

Gastric cancer is prone to metastasis. ${ }^{10}$ Research shows that following gastrectomy in GC patients, about $2.43 \%$ of cases have liver metastasis. ${ }^{11}$ The lung, bone and brain metastasis rates of GC patients are $0.96 \%,{ }^{12} 6.7 \%{ }^{13}$ and $2.33 \%,{ }^{14}$ respectively. Relatively few patients with GC have ocular metastasis (OM), and their main ocular manifestations are eyelid swelling, eye pain and even blindness. ${ }^{15}$ Despite successful ocular treatment, life prognosis is poor. ${ }^{16}$ In order to alleviate the patients' eye symptoms and pain, the early detection and diagnosis of
$\mathrm{OM}$ is of great significance for early intervention and better prognosis.

Studies have shown that INcRNA MEG 3 can inhibit the proliferation and metastasis of GC through the p53 signaling pathway, ${ }^{17}$ and that downregulation of ARK5 gene expression can inhibit the invasion and metastasis of GC. ${ }^{18}$ In addition, the transfection of miRNA-21 inhibitor can downregulate the expression of miRNA-21, resulting in the downregulation of epithelial-to-mesenchymal transition and the inhibition of GC cell invasion and migration. ${ }^{19}$ However, the above methods are difficult to implement, so they are rarely used in clinical practice. Serological markers exist in tumor cells and have unique advantages in the diagnosis of GC metastasis due to their easy access. They can be used as clinical routine detection indicators to assist in the diagnosis of tumor metastasis. Tumor markers refer to the substances that can be detected in blood, body fluid and tissue, which are produced abnormally by tumor cells or induced by the host's stimulation response to tumor. The immunological characteristics of these substances make it possible to detect tumor and gauge its development, prognosis and effectiveness of therapy. ${ }^{20}$ In the present study we investigated whether serological and tumor markers can be used to differentiate OM in patients with gastric adenocarcinoma. We also analyzed the correlations between $\mathrm{OM}$ and the risk factors for gastric adenocarcinoma, to find accurate indicators for OM in patients with gastric adenocarcinoma. The overarching aims of the study were to facilitate early diagnosis and intervention for metastases to provide a basis for estimates of prognosis, and to improve the quality of life and remission rate of patients with gastric adenocarcinoma.

\section{Patients and Methods \\ Study Design}

This study included patients diagnosed with gastric adenocarcinoma between June 6, 2003 to July 8, 2019, and excluded other types of gastric cancer. The diagnosis was confirmed by pathological examination. Patients who developed eye metastases of gastric adenocarcinoma, diagnosed by imaging and histology, were categorized as ocular metastasis (OM) patients. All others were categorized as non-ocular metastasis (NOM). All the subjects received information about the purpose and methods of the study before agreeing to participate in the trial and signing a declaration of informed consent. 


\section{Data Collection}

Data were collected from patients' records, including demographic data (gender, age and histological grading) and the following serological test results at the time of diagnosis: hemoglobin (HB), calcium, alkaline phosphatase (ALP), total cholesterol (TC), triglyceride (TG), high-density lipoprotein (HDL), low-density lipoprotein (LDL), apolipoprotein A1 (ApoA1), apolipoprotein B (ApoB), alpha fetoprotein (AFP), carbohydrate antigen-724 (CA724), carcinoembryonic antigen (CEA), CA125, CA153, CA199, cytokeratin-19 fragment (CYFRA21-1), lipoprotein(a) (Lp(a)).

\section{Statistical Analyses}

Gender and histological grade were compared between the two groups using a chi square test, and serological indices were compared using an independent samples $t$-test. A binary logistic regression model was used to analyze the significance of serum markers in $\mathrm{OM}$ of patients with gastric adenocarcinoma. Finally, ROC curves were used to analyze the significance of relevant indicators in the identification of OM. SPSS 24.0 software and excel 2017 software were used in this data analysis. P values of $<0.05$ were considered significant.

\section{Results}

\section{Demographics and Clinical Characteristics of Participants}

In total, 3056 patients with gastric adenocarcinoma were included, with 22 patients in the OM group (19 males and 3 females) and 3034 patients in the NOM group (2009 males and 1025 females). A significant difference in gender composition was found between the two groups ( $\mathrm{P}<$ $0.05)$. Mean age was statistically similar in the OM $(61.55$ \pm 9.96 years), and NOM group $(56.20 \pm 13.04$ years; $\mathrm{P}>$ $0.05)$. No significant difference in histological grade was found between the two groups $(\mathrm{P}>0.05)$. See Table 1 and Figure 1Figure 2Figure -3 for specific clinical information and relevant fundus examination results.

\section{Correlation Analysis of Serological Indices Between the Two Groups}

Levels of LDL and CA724 were significantly higher in the OM than NOM group $(\mathrm{P}<0.05)$. However, $\mathrm{Hb}$, calcium, ALP, TC, TG, HDL, ApoA1, ApoB, AFP, CA199, CA125, CA153, CYFRA21-1 and Lp(a) were similar in the two groups $(\mathrm{P}>0.05)$. In addition, binary logistic regression analysis showed that LDL was an independent risk factor for OM. See Tables 2 and 3 for details.

\section{ROC Analysis of LDL and CA724 as Factors in the Diagnosis of OM}

Critical values of LDL and CA724 were $3.67 \mathrm{mmol} / \mathrm{L}$ and $13.02 \mathrm{U} / \mathrm{mL}$, respectively. In other words, when the LDL levels are higher than $3.67 \mathrm{mmol} / \mathrm{L}$ or the CA724 levels are higher than $13.02 \mathrm{U} / \mathrm{mL}$, patients with gastric adenocarcinoma are more likely to have OM. The AUC of LDL was 0.903 (95\% CI: $0.562-0.739)$ and the sensitivity and specificity were $91 \%$ and $82 \%$, respectively $(p<0.001$ ). The AUC of CA724 was 0.913 (95\% CI: 0.984-1.000) and the sensitivity and specificity were $100 \%$ and $86 \%$, respectively ( $\mathrm{p}<0.001)$. The AUC of CA724 was higher than that of LDL, indicating that CA724 has higher diagnostic accuracy. The ROC curve of OM risk factors in patients with gastric adenocarcinoma is shown in Figure 4A. The AUC for LDL/CA724 ratio and the combined indicators of LDL + CA724 were calculated as potential risk factors for OM. The AUC value of LDL + CA724 was as high as 0.934 (see Figure 4B for details) $(\mathrm{p}<0.001)$. The sensitivity of CA724, LDL/CA724 and LDL + CA724 was 1.00 in each case, and the specificity of LDL + CA724 was 0.87. See Table 4 for specific data.

\section{Discussion}

We analyzed 3056 patients with gastric adenocarcinoma and studied their serological indices and risk factors for $\mathrm{OM}$ in detail. The incidence rate of gastric cancer is closely related to age and is particularly high in patients 80 years of age or over. ${ }^{21}$ In this study, the male to female ratio of gastric adenocarcinoma was 1.97:1, with a higher proportion of male than female patients. Studies have shown that the high pressure of life and work continuously stimulates the cerebral cortex, and lesions caused by the continuous excitation of sympathetic nerves may be high risk factors for men. ${ }^{22}$ At present, the main causes of gastric adenocarcinoma are Helicobacter pylori infection, poor dietary habits and genetic factors. Among the patients involved in this study, $93.4 \%$ had poorly or moderately differentiated adenocarcinoma. Poor differentiation occurred with a high degree of malignancy and poorer prognosis.

Most patients with early gastric adenocarcinoma have no distinct symptoms or signs, so may not be concerned at this stage. At an advanced stage, patients are admitted to 
Table I The Clinical Characteristics in OM Group and NOM Group

\begin{tabular}{|c|c|c|c|}
\hline Patient Characteristics & OM Group $^{a}(n=22)(0.7 \%)$ & NOM Group $(n=3034)(99.3 \%)$ & P value ${ }^{d}$ \\
\hline \multicolumn{4}{|l|}{ Gender ${ }^{b}$} \\
\hline Male & 19 & 2009 & 0.046 \\
\hline Female & 3 & 1025 & \\
\hline \multicolumn{4}{|l|}{$\mathrm{Age}^{\mathrm{c}}$} \\
\hline Mean & $61.55 \pm 9.96$ & $56.20 \pm 13.04$ & 0.055 \\
\hline \multicolumn{4}{|l|}{ Histological grading ${ }^{\mathrm{b}}$} \\
\hline Poorly differentiated adenocarcinoma & 22 & 1769 & 0.001 \\
\hline Moderately differentiated adenocarcinoma & 0 & 1062 & \\
\hline Well-differentiated adenocarcinoma & 0 & 72 & \\
\hline Others & 0 & $|3|$ & \\
\hline \multicolumn{4}{|l|}{ Transfer position ${ }^{\mathrm{b}}$} \\
\hline Hepatic metastases & 11 & 364 & 0.001 \\
\hline Bone metastases & 19 & 54 & \\
\hline Pulmonary metastases & 2 & 38 & \\
\hline Splenic metastases & $\mathrm{I}$ & 1 & \\
\hline Brain metastases & 4 & 2 & \\
\hline Lymph node metastases & 3 & 204 & \\
\hline Peritoneal Metastases & 0 & 270 & \\
\hline Others & 0 & 253 & \\
\hline \multicolumn{4}{|l|}{ Treatment $^{\mathrm{b}}$} \\
\hline Palliative surgery & 2 & 126 & 0.475 \\
\hline Radical gastrectomy for gastric cancer & 20 & 2386 & \\
\hline Chemotherapy & 21 & $217 \mid$ & \\
\hline Supportive treatment & 0 & 179 & \\
\hline
\end{tabular}

Notes: Data showed as mean \pm standard deviation or $\mathrm{n} . \mathrm{P}<0.05$ indicates statistical significance. ${ }^{\mathrm{a}} \mathrm{OM}$, including intraocular metastasis and eyelid metastasis. ${ }^{\mathrm{b}} \mathrm{Chi}-\mathrm{squared}$ test. 'Student's $t$-test. 'Comparison between the OM and NOM groups.

hospital for fever, severe pain, significant weight loss, hematemesis, or metastasis symptoms. At present, gastric adenocarcinoma is the most common type of gastric cancer. Advanced gastric cancer is prone to metastasis, and its prognosis is often poor. Therefore, many studies have looked for predictors of metastasis by studying risk factors. Several such studies have been conducted in recent years, and the details of these are shown in Table $5 .^{21-28}$

Ocular metastases of gastric cancer manifest as eyelid swelling, eye pain and even blindness ${ }^{15}$ and have poor prognosis with high mortality. ${ }^{16}$ Ocular metastases are fortunately very rare in gastric cancer, occurring more commonly in breast and lung cancer. ${ }^{29}$ A Japanese study shows that, when ocular metastases do occur in gastric cancer, the average time from the definitive primary diagnosis to the occurrence of eye symptoms is 25.4 months, and the average time from the occurrence of those symptoms to death is only 3.3 months. ${ }^{30}$ Therefore, early detection and diagnosis of OM is of great significance for early intervention and better prognosis. Since some eye metastases are relatively hidden and may be misdiagnosed as other diseases, it is important to improve detection by fully combining the patient's history and relevant examination results. Unfortunately, early identification of gastric cancer metastasis remains challenging in practice. The clinical use of computed tomography and magnetic resonance imaging methods is not extensive due to high cost and limited sensitivity and specificity. Therefore, low price and convenient serum tumor markers have been widely used in clinical practice.

In the present study, LDL and CA724 were significantly lower in patients with than without ocular metastases. Analysis showed that LDL concentration may be used as an independent risk factor for the differential diagnosis of OM. Further analysis showed that LDL and CA724 may be important risk factors for OM in patients with gastric adenocarcinoma and identified threshold concentrations at which gastric adenocarcinoma patients are at 


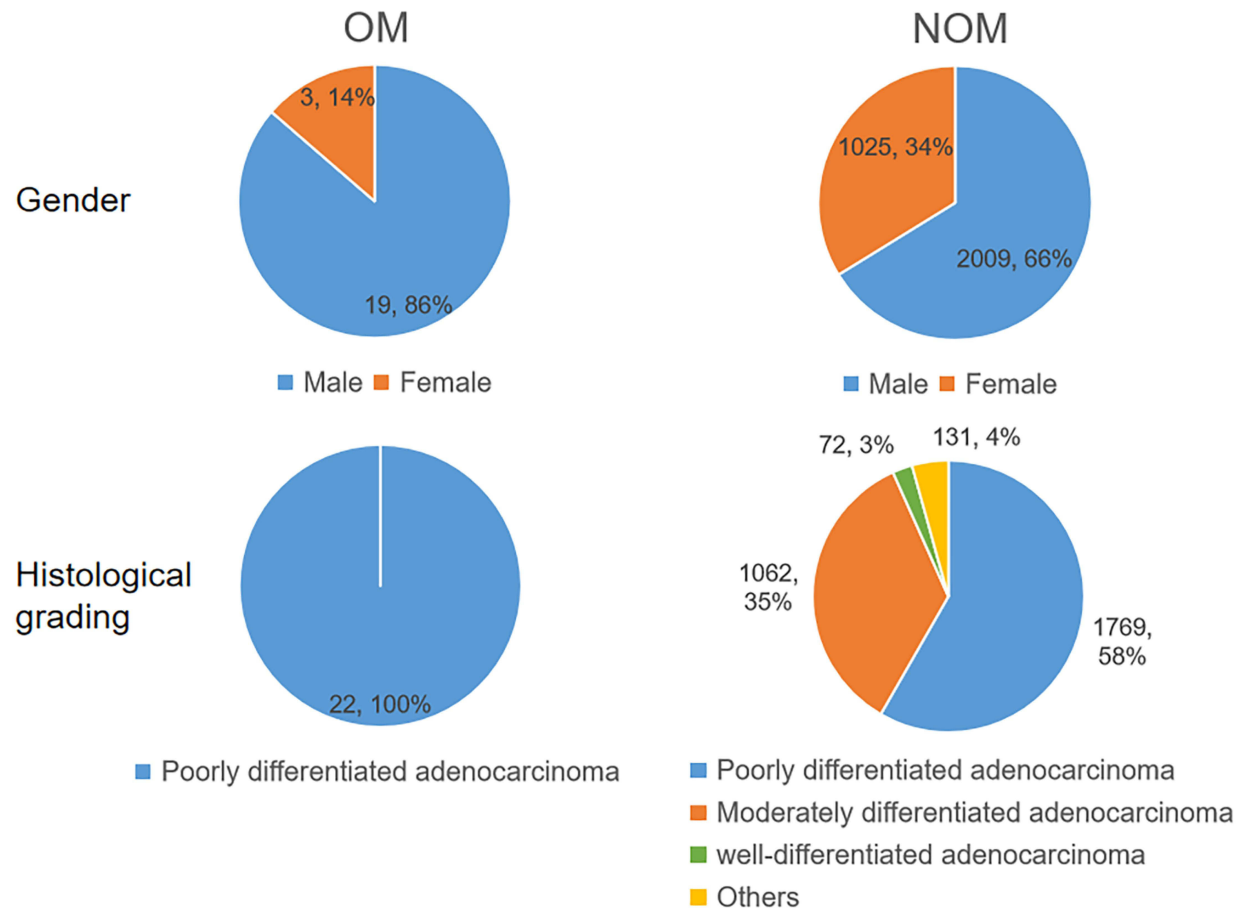

Figure I Clinical characteristics of gastric adenocarcinoma OM patients and NOM patients.

Note: $n=22$ in OM group, $n=3034$ in NOM group.

Abbreviations: OM, ocular metastasis; NOM, non-ocular metastasis.

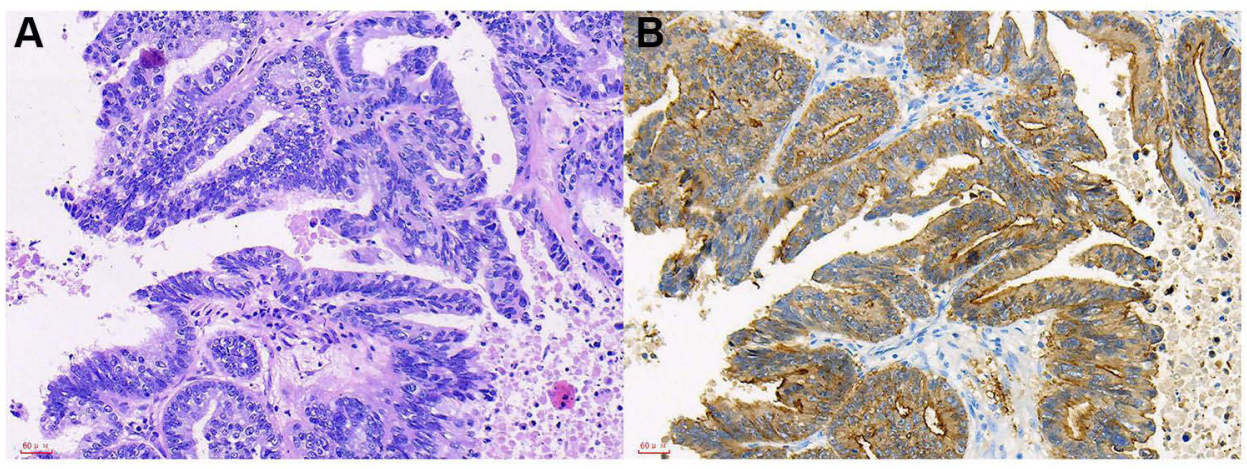

Figure 2 The HE staining and IHC images from gastric adenocarcinoma OM patients.

Notes: (A) gastric adenocarcinoma (HE×200). (B) Villin (+) (SP×200). The tissue was collected from OM site of gastric adenocarcinoma patients. Abbreviations: HE, hematoxylin-eosin; IHC, immunohistochemistry; OM, ocular metastasis; SP, streptavidin-peroxidase.

risk of OM. A combination of these risk factors can further improve the differential diagnosis of $\mathrm{OM}$, thus enabling earlier treatment and improved prognosis.

Low density lipoprotein (LDL) is a form of lipoprotein particle. Antibodies against oxidized LDL can be found in atherosclerotic lesions. When LDL and oxidized LDL exist, cellular biochemistry and signaling pathways can produce pro atherosclerotic changes, and antioxidants in vitro can reduce or reverse the development of atherosclerosis. ${ }^{31}$ Studies have shown that LDL receptor associated protein can promote the invasion of human breast cancer cells in vitro. ${ }^{32}$ LDL can also be used as a prognostic indicator for patients with small cell lung cancer. ${ }^{33}$ In addition, the analysis of serum markers showed that preoperative LDL was an independent risk factor for recurrence of prostate cancer, ${ }^{34}$ and an independent prognostic factor for colorectal cancer. ${ }^{35}$ Unhealthy diet, obesity, mental stress and genetic factors can lead to high levels of LDL. ${ }^{36}$ In our study, LDL content was significantly increased in patients with gastric 


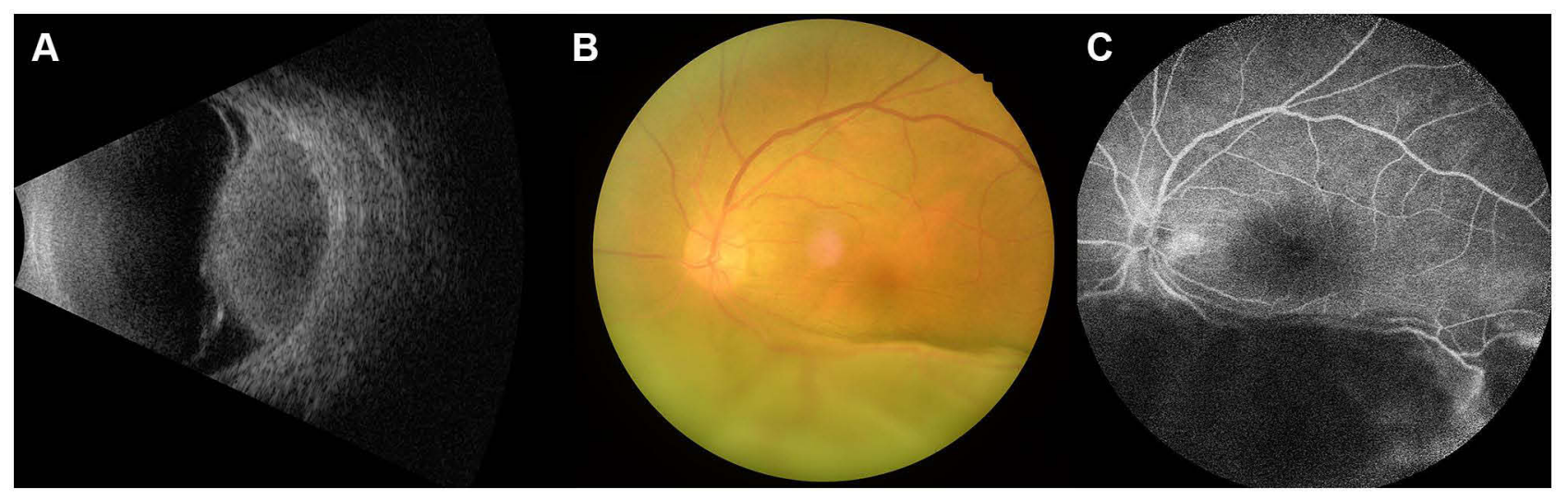

Figure 3 Imaging and pathological data of OM in gastric adenocarcinoma patients.

Notes: (A) Ultrasound examination of the eye. The relative shape of the hypoechoic mass is seen. (B) Fundus photography of an OM patient with gastric adenocarcinoma. (C) Fluorescence leakage and low fluorescence were observed in the lesions.

Abbreviation: OM, ocular metastasis.

adenocarcinoma, and it can be used as an independent risk factor for the differential diagnosis of OM. It seems feasible that LDL may promote gastrointestinal inflammation through activating reactive oxygen species and mitogenactivated protein kinase signaling pathways ${ }^{37}$ and may thus increase the risk of cancer.

CA724 is a molecular glycoprotein, mainly distributed in the stomach, breast, pancreas and ovary. It is a tumor marker mainly used to detect gastric cancer and various digestive tract cancers. A 2012 study showed that CA724

Table 2 Differences in the Concentration of Various Tumor Biomarkers Between OM and NOM

\begin{tabular}{|c|c|c|c|c|}
\hline $\begin{array}{l}\text { Tumor } \\
\text { Biomarkers }\end{array}$ & OM Group & NOM Group & $t$-test & P-value \\
\hline $\mathrm{HB}(\mathrm{g} / \mathrm{L})$ & $119.09 \pm 16.98$ & $114.55 \pm 21.84$ & 0.973 & 0.331 \\
\hline Calcium (mmol/L) & $2.24 \pm 0.25$ & $2.23 \pm 0.26$ & 0.261 & 0.794 \\
\hline ALP $(U / L)$ & $103.82 \pm 86.28$ & $115.00 \pm \mid 18.82$ & -0.441 & 0.660 \\
\hline TC (mmol/l) & $7.86 \pm 16.15$ & $4.68 \pm 1.57$ & 0.925 & 0.366 \\
\hline TG (mmol/L) & $1.26 \pm 0.62$ & $1.65 \pm 1.33$ & -1.346 & 0.178 \\
\hline $\mathrm{HDL}(\mathrm{mmol} / \mathrm{L})$ & $1.53 \pm 0.72$ & $1.66 \pm 1.23$ & -0.483 & 0.629 \\
\hline LDL (mmol/L) & $5.19 \pm 1.20$ & $2.92 \pm 1.42$ & 7.492 & 0.000 \\
\hline ApoAI (g/L) & $1.45 \pm 0.32$ & $1.67 \pm 1.02$ & -0.982 & 0.326 \\
\hline ApoB (g/L) & $1.37 \pm 1.19$ & $1.16 \pm 0.79$ & 1.227 & 0.220 \\
\hline $\operatorname{AFP}(n g / m L)$ & $3.65 \pm 1.95$ & $5.05 \pm 35.88$ & -0.182 & 0.855 \\
\hline CEA (ng/mL) & $16.82 \pm 9.61$ & $13.34 \pm 93.33$ & 0.175 & 0.861 \\
\hline CA724 (U/mL) & $16.61 \pm 2.89$ & $8.31 \pm 17.22$ & 2.260 & 0.024 \\
\hline CAI25 (U/mL) & $68.75 \pm 117.43$ & $53.80 \pm 133.28$ & 0.525 & 0.600 \\
\hline CAI53 (U/mL) & $14.11 \pm 12.65$ & $16.49 \pm 21.68$ & -0.515 & 0.607 \\
\hline CAI99 (U/mL) & $28.20 \pm 46.78$ & $72.04 \pm 341.94$ & -0.601 & 0.548 \\
\hline CYFRA2I-I (ng/mL) & $3.20 \pm 1.53$ & $5.73 \pm 11.34$ & -1.046 & 0.295 \\
\hline $\operatorname{Lp}(\mathrm{A})(\mathrm{mg} / \mathrm{L})$ & $224.03 \pm 145.32$ & $212.96 \pm 169.16$ & 0.306 & 0.760 \\
\hline
\end{tabular}

Notes: Independent sample t-test. $\mathrm{P}<0.05$ denoted statistical significance. Data showed as mean \pm standard deviation.

Abbreviations: OM, ocular metastasis; NOM, non-ocular metastasis. is the most relevant serum tumor marker in Chinese patients with gastric cancer. ${ }^{38}$ CA725 concentration was positively correlated with tumor stage, recurrence, distant metastasis and death risk, and correlation analysis showed that CA725 concentration level was a significant predictor for sensitivity to chemotherapy. ${ }^{39}$ These findings together with those of the present study suggest that the prognosis in $\mathrm{OM}$ is poor, the condition may readily relapse, and most cancer cells may have metastasized, so the survival rate in $\mathrm{OM}$ is low.

We found that when LDL + CA724 were analyzed together, the AUC of ROC curve was higher than when these factors were considered alone, indicating high sensitivity and specificity. These findings suggest that this combination of factors may be of value in predicting OM in gastric adenocarcinoma. In addition, it is also conducive to our early eye intervention and treatment to prevent the further deterioration of the disease and reduce the suffering of patients, so as to improve the quality of life of patients.

It is worth noting that this study has some limitations. First, the data may have been affected by recall bias. In addition, due to the low incidence of eye metastases in gastric

Table 3 Risk Factors of OM in Patients with Gastric Adenocarcinoma

\begin{tabular}{|l|l|r|r|r|}
\hline Tumor Marker & B & Exp(B) & OR $(\mathbf{9 5} \% \mathbf{C l})$ & P value \\
\hline LDL & -0.439 & 0.645 & $0.562-0.739$ & 0.000 \\
CA724 & -0.008 & 0.992 & $0.984-1.000$ & 0.053 \\
\hline
\end{tabular}

Notes: Binary logistic regression analysis. $\mathrm{P}<0.05$ denoted statistical significance. Abbreviations: $\mathrm{B}$, coefficient of regression; $\mathrm{OR}$, odds ratio; $\mathrm{Cl}$, confidence interval; OM, ocular metastasis; LDL, low-density lipoprotein; CA724, carbohydrate antigen-724. 

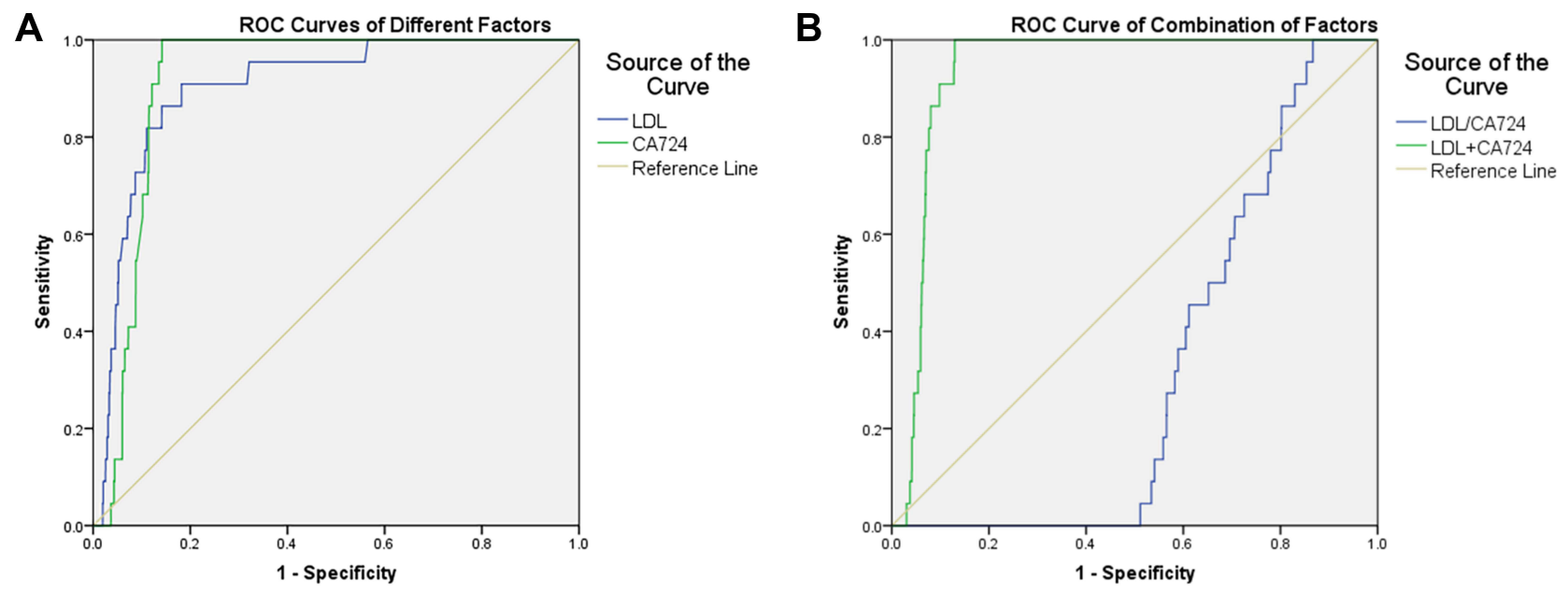

Figure 4 The receiver operating characteristic (ROC) curves of risk factors for detecting $O M$ in gastric adenocarcinoma patients.

Notes: (A) ROC curves of LDL and CA724 as single risk factors for OM. (B) ROC curves of LDL to CA724 ratio and LDL+CA724.

Abbreviations: OM, ocular metastasis; LDL, low-density lipoprotein; CA724, carbohydrate antigen-724.

adenocarcinoma, even though we included 3056 patients with gastric adenocarcinoma, only 22 patients were included in the OM group. A larger sample of patients in this category would increase statistical power and confidence in the results. In addition, all the patients were from the same hospital, so the analysis may not represent the whole population of patients with $\mathrm{OM}$ in gastric adenocarcinoma.

\section{Conclusions}

The results of this study indicate that LDL and CA724 are risk factors for $\mathrm{OM}$ in gastric adenocarcinoma. Importantly, the combination of LDL and CA724 has value in more accurately predicting the occurrence of eye metastases in patients with gastric adenocarcinoma. These indicators may allow timely diagnosis of $\mathrm{OM}$ in patients

Table 4 The Cut-off Value, Sensitivity, Specificity, and AUC of Risk Factors for the Prediction of OM in Patients

\begin{tabular}{|l|c|c|c|c|c|}
\hline Factor & Cut-Off Value & Sensitivity (\%) & Specificity (\%) & AUC & P value \\
\hline LDL $(\mathrm{mmol} / \mathrm{L})$ & 3.67 & 0.91 & 0.82 & 0.903 & $<0.001$ \\
CA724 $(\mathrm{U} / \mathrm{mL})$ & 13.02 & 1.00 & 0.86 & 0.913 & $<0.001$ \\
LDL/CA724 & 0.17 & 1.00 & 0.13 & 0.325 & $<0.005$ \\
LDL+CA724 & - & 1.00 & 0.87 & 0.934 & $<0.001$ \\
\hline
\end{tabular}

Notes: Sensitivity and specificity were acquired at the cut-off value. $\mathrm{P}<0.05$ denoted statistical significance.

Abbreviations: AUC, area under the curve; OM, ocular metastasis; LDL, low-density lipoprotein; CA724, carbohydrate antigen-724.

Table 5 The Risk Factors of Metastases of Gastric Cancer

\begin{tabular}{|l|l|l|l|}
\hline Author & Year & Metastatic Sites & Risk Factor \\
\hline Liu et al $^{21}$ & 2015 & Lymphoid node & Claudin-4 \\
Asaka et al $^{22}$ & 2017 & Lymphoid node & CAI99 \\
Marques-Lespier et al $^{23}$ & 2017 & Peritoneal metastasis & CAI25, CA724 \\
Chen et al $^{24}$ & 2019 & NS & THBS4 \\
Ji et al & 2019 & NS & LINC00086, miR-214 \\
Kong et al $^{26}$ & 2019 & Lymphoid node & MiR-25 \\
Miwa et al $^{27}$ & 2019 & Hepatic and peritoneal & HOXCI0 \\
Jing et al & 2020 & Lymphoid node & CEA, CAI99 \\
\hline
\end{tabular}

Abbreviations: NS, not specific; THBS4, thrombospondin 4; CEA, carcinoembryonic antigen; CAI25, carbohydrate antigen-125; CA724, carbohydrate antigen-724; CAI99, carbohydrate antigen-199; HOXCI0, homeobox Cl0. 
with gastric adenocarcinoma, allow early intervention, and improve the prognosis of patients with this condition.

\section{Data Sharing Statement}

We are willing to share our data information. Relevant data information can be obtained by contacting the corresponding author.

\section{Ethics Approval and Informed Consent}

This study has been reviewed and approved by the medical ethics committee of the First Affiliated Hospital of Nanchang University, and strictly abides by the Helsinki declaration. After knowing the significance of the study, all the patients kept a positive attitude and signed the informed consent one by one.

\section{Acknowledgments}

We thank the patients who participated in this study for their medical records.

\section{Funding}

This research is supported by Key Research Foundation of Jiangxi Province (No: 20203BBG73059, 20181BBG70004); Excellent Talents Development Project of Jiangxi Province (No: 20192BCBL23020); Natural Science Foundation of Jiangxi Province (No: 20181BAB205034); Grassroots Health Appropriate Technology "Spark Promotion Plan" Project of Jiangxi Province (No: 20188003); Health Development Planning Commission Science Foundation of Jiangxi Province (No: 20201032); Health Development Planning Commission Science TCM Foundation of Jiangxi Province (No: 2018A060).

\section{Disclosure}

The authors declare that they have no competing interests.

\section{References}

1. Smyth EC, Nilsson M, Grabsch HI, van Grieken NC, Lordick F. Gastric cancer. Lancet. 2020;396(10251):635-648.

2. Jemal A, Center MM, DeSantis C, Ward EM. Global patterns of cancer incidence and mortality rates and trends. Cancer Epidemiol Biomarkers Prev. 2010;19(8):1893-1907. doi:10.1158/1055-9965.EPI-10-0437

3. Rima FA, Hussain M, Dewan RK, et al. Clinicopathologic features of gastric and gastrooesophageal junction adenocarcinoma. Mymensingh Med J. 2020;29(1):195-201.

4. Karimi P, Islami F, Anandasabapathy S, Freedman ND, Kamangar F. Gastric cancer: descriptive epidemiology, risk factors, screening, and prevention. Cancer Epidemiol Biomarkers Prev. 2014;23(5):700-713. doi:10.1158/1055-9965.EPI-13-1057
5. Kamangar F, Sheikhattari P, Mohebtash M. Helicobacter pylori and its effects on human health and disease. Arch Iran Med. 2011;14(3):192-199.

6. Chiba T, Marusawa H, Seno H, Watanabe N. Mechanism for gastric cancer development by Helicobacter pylori infection. $J$ Gastroenterol Hepatol. 2008;23(8 Pt 1):1175-1181. doi:10.1111/j.1440-1746.2008.05472.x

7. Correa P. Gastric cancer: overview. Gastroenterol Clin North Am. 2013;42(2):211-217. doi:10.1016/j.gtc.2013.01.002

8. Das M. Neoadjuvant chemotherapy: survival benefit in gastric cancer. Lancet Oncol. 2017;18(6):e307. doi:10.1016/S1470-2045(17)30321-2

9. Tan Z. Recent advances in the surgical treatment of advanced gastric cancer: a review. Med Sci Monit. 2019;25:3537-3541. doi:10.12659/ MSM.916475

10. Zhao X, Li X, Yuan H. microRNAs in gastric cancer invasion and metastasis. Front Biosci. 2013;18(3):803-810. doi:10.2741/4144

11. Markar SR, Mackenzie H, Mikhail S, et al. Surgical resection of hepatic metastases from gastric cancer: outcomes from national series in England. Gastric Cancer. 2017;20(2):379-386. doi:10.1007/ s10120-016-0604-6

12. Kong JH, Lee J, Yi CA, et al. Lung metastases in metastatic gastric cancer: pattern of lung metastases and clinical outcome. Gastric Cancer. 2012;15(3):292-298. doi:10.1007/s10120-011-0104-7

13. Kim YJ, Kim SH, Kim JW, et al. Gastric cancer with initial bone metastasis: a distinct group of diseases with poor prognosis. Eur $J$ Cancer. 2014;50(16):2810-2821. doi:10.1016/j.ejca.2014.08.003

14. Cavanna L, Seghini P, Di Nunzio C, et al. Gastric cancer with brain metastasis and the role of human epidermal growth factor 2 status. Oncol Lett. 2018;15(4):5787-5791.

15. Tsuruta Y, Maeda Y, Kitaguchi Y, et al. A case of endonasal endoscopic surgery for intraorbital metastasis of gastric ring cell carcinoma. Ear Nose Throat J. 2020;145561320943372.

16. Celebi AR, Kilavuzoglu AE, Altiparmak UE, Cosar CB, Ozkiris A. Iris metastasis of gastric adenocarcinoma. World J Surg Oncol. 2016;14(1):71. doi:10.1186/s12957-016-0840-6

17. Wei GH, Wang X. IncRNA MEG3 inhibit proliferation and metastasis of gastric cancer via p53 signaling pathway. Eur Rev Med Pharmacol Sci. 2017;21(17):3850-3856.

18. Chen D, Liu G, Xu N, et al. Knockdown of ARK5 expression suppresses invasion and metastasis of gastric cancer. Cell Physiol Biochem. 2017;42(3):1025-1036. doi:10.1159/000478685

19. Xiao T, Jie Z. MiR-21 promotes the invasion and metastasis of gastric cancer cells by activating epithelial-mesenchymal transition. Eur Surg Res. 2019;60(5-6):208-218. doi:10.1159/000504133

20. Faria SC, Sagebiel T, Patnana M, et al. Tumor markers: myths and facts unfolded. Abdom Radiol. 2019;44(4):1575-1600. doi:10.1007/ s00261-018-1845-0

21. Liu JX, Wei ZY, Chen JS, Lu HC, Hao L, Li WJ. Prognostic and clinical significance of claudin-4 in gastric cancer: a meta-analysis. World J Surg Oncol. 2015;13(1):207. doi10.1186/s12957-015-0626-2

22. Asaka M, Kobayashi M, Kudo T, et al. Gastric cancer deaths by age group in Japan: outlook on preventive measures for elderly adults. Cancer Sci. 2020;111(10):3845-3853. doi:10.1111/cas.14586

23. Marqués-Lespier JM, González-Pons M, Cruz-Correa M. Current perspectives on gastric cancer. Gastroenterol Clin North Am. 2016;45(3):413-428. doi:10.1016/j.gtc.2016.04.002

24. Chen X, Huang Y, Wang Y, Wu Q, Hong S, Huang Z. THBS4 predicts poor outcomes and promotes proliferation and metastasis in gastric cancer. J Physiol Biochem. 2019;75(1):117-123. doi:10.1007/ s13105-019-00665-9

25. Ji B, Huang Y, Gu T, Zhang LE, Li G, Zhang C. Potential diagnostic and prognostic value of plasma long noncoding RNA LINC00086 and miR-214 expression in gastric cancer. Cancer Biomark. 2019;24 (2):249-255. doi:10.3233/CBM-181486

26. Kong Y, Ning L, Qiu F, Yu Q, Cao B. Clinical significance of serum miR-25 as a diagnostic and prognostic biomarker in human gastric cancer. Cancer Biomark. 2019;24(4):477-483. doi:10.3233/CBM182213 
27. Miwa T, Kanda M, Umeda S, et al. Homeobox C10 influences on the malignant phenotype of gastric cancer cell lines and its elevated expression positively correlates with recurrence and poor survival. Ann Surg Oncol. 2019;26(5):1535-1543. doi:10.1245/s10434-01907166-5

28. Jing R, Cui M, Ju S, Pan S. The changes and clinical significance of preoperative and postoperative serum CEA and CA19-9 in Gastric Cancer. Clin Lab. 2020;66(4). doi:10.7754/Clin.Lab.2019.190732

29. Goto S, Takeda H, Sasahara Y, Takanashi I, Yamashita H. Metastasis of advanced gastric cancer to the extraocular muscle: a case report. J Med Case Rep. 2019;13(1):107. doi:10.1186/s13256-019-2031-x

30. Amemiya T, Hayashida H, Dake Y. Metastatic orbital tumors in Japan: a review of the literature. Ophthalmic Epidemiol. 2002;9 (1):35-47. doi:10.1076/opep.9.1.35.1718

31. Hamilton CA. Low-density lipoprotein and oxidised low-density lipoprotein: their role in the development of atherosclerosis. Pharmacol Ther. 1997;74(1):55-72. doi:10.1016/S0163-7258(96) 00202-1

32. Li Y, Wood N, Grimsley P, Yellowlees D, Donnelly PK. In vitro invasiveness of human breast cancer cells is promoted by low density lipoprotein receptor-related protein. Invasion Metastasis. 1998;18(56):240-251. doi:10.1159/000024517

33. Zhou T, Zhan J, Fang W, et al. Serum low-density lipoprotein and low-density lipoprotein expression level at diagnosis are favorable prognostic factors in patients with small-cell lung cancer (SCLC). BMC Cancer. 2017;17(1):269. doi:10.1186/s12885-017-3239-Z
34. Wettstein MS, Saba K, Umbehr MH, et al. Prognostic role of preoperative serum lipid levels in patients undergoing radical prostatectomy for clinically localized prostate cancer. Prostate. 2017;77 (5):549-556. doi:10.1002/pros.23296

35. Liu YL, Qian HX, Qin L, Zhou XJ, Zhang B. Serum LDL-C and LDL-C/HDL-C ratio are positively correlated to lymph node stages in males with colorectal cancer. Hepatogastroenterology. 2011;58 (106):383-387.

36. Krauss RM. Dietary and genetic effects on low-density lipoprotein heterogeneity. Annu Rev Nutr. 2001;21(1):283-295. doi:10.1146/ annurev.nutr.21.1.283

37. Wang C, Li P, Xuan J, et al. Cholesterol enhances colorectal cancer progression via ROS elevation and MAPK signaling pathway activation. Cell Physiol Biochem. 2017;42(2):729-742. doi:10.1159/ 000477890

38. Chen XZ, Zhang WK, Yang K, et al. Correlation between serum CA724 and gastric cancer: multiple analyses based on Chinese population. Mol Biol Rep. 2012;39(9):9031-9039. doi:10.1007/ s11033-012-1774-X

39. Zou L, Qian J. Decline of serum CA724 as a probable predictive factor for tumor response during chemotherapy of advanced gastric carcinoma. Chin J Cancer Res. 2014;26(4):404-409.

\section{Publish your work in this journal}

Cancer Management and Research is an international, peer-reviewed open access journal focusing on cancer research and the optimal use of preventative and integrated treatment interventions to achieve improved outcomes, enhanced survival and quality of life for the cancer patient.
The manuscript management system is completely online and includes a very quick and fair peer-review system, which is all easy to use. Visit http://www.dovepress.com/testimonials.php to read real quotes from published authors. 